\title{
Rapid rotation of micron and submicron dielectric particles measured using optical tweezers
}

\author{
ALEXANDER D. ROWE ${ }^{\dagger}$, MARK C. LEAKE ${ }^{\dagger}$, \\ HYWEL MORGAN ${ }^{\dagger \ddagger}$ and RICHARD M. BERRY ${ }^{\dagger *}$ \\ ${ }^{\dagger}$ The Clarendon Laboratory, Department of Physics, \\ Oxford University, Parks Road, Oxford OX1 3PU, UK; \\ e-mail: r.berry1@physics.ox.ac.uk \\ ${ }^{\ddagger}$ Department of Electrical and Electronic Engineering, \\ University of Glasgow, Glasgow G12 8QQ, UK
}

(Received 27 August 2002; revision received 31 October 2002)

\begin{abstract}
We demonstrate the use of a laser trap ('optical tweezers') and back-focal-plane position detector to measure rapid rotation in aqueous solution of single particles with sizes in the vicinity of $1 \mu \mathrm{m}$. Two types of rotation were measured: electrorotation of polystyrene microspheres and rotation of the flagellar motor of the bacterium Vibrio alginolyticus. In both cases, speeds in excess of $1000 \mathrm{~Hz}\left(\mathrm{rev} \mathrm{s}^{-1}\right)$ were measured. Polystyrene beads of diameter about $1 \mu \mathrm{m}$ labelled with smaller beads were held at the centre of a microelectrode array by the optical tweezers. Electrorotation of the labelled beads was induced by applying a rotating electric field to the solution using microelectrodes. Electrorotation spectra were obtained by varying the frequency of the applied field and analysed to obtain the surface conductance of the beads. Single cells of $V$. alginolyticus were trapped and rotation of the polar sodium-driven flagellar motor was measured. Cells rotated more rapidly in media containing higher concentrations of $\mathrm{Na}^{+}$, and photodamage caused by the trap was considerably less when the suspending medium did not contain oxygen. The technique allows single-speed measurements to be made in less than a second and separate particles can be measured at a rate of several per minute.
\end{abstract}

\section{Introduction}

The technique of optical tweezers uses a focused laser beam to trap small dielectric particles in three dimensions [1]. The laser beam is brought to a diffraction-limited focus by a high-numerical-aperture (NA) (greater than unity) microscope objective lens. Particles with a refractive index greater than their surroundings (typically an aqueous solution) are drawn into what is essentially a potential well at the focal point. This may be understood either by considering the exchange of momentum between a trapped particle and the incident photons which it deflects, or in terms of minimization of the energy in the laser radiation field. Trapping forces have been modelled using ray optics and Rayleigh scattering for particles much larger or much smaller than respectively the wavelength of the trapping laser beam [2]. With a laser power in the range of $100 \mathrm{~mW}$ in the near

*Author for correspondence. 
infrared, typical trapping forces on polystyrene particles about $1 \mu \mathrm{m}$ in diameter in aqueous solution are in the range of several piconewtons and trap stiffness is up to approximately $0.1 \mathrm{pN} \mathrm{nm}^{-1}$. These values are ideal for the study of biological molecular motors, and optical tweezers have been used to measure the piconewton forces and nanometre displacements generated by several different molecular motors [3]. Forces may be calibrated using Stokes drag, while displacements may be measured from the image of the trapped particle [4], or alternatively by measuring the deflection of either the trapping beam $[5,6]$ or a second separate laser beam [7]. In the latter technique, the emerging laser beam is collimated by a condenser lens and the back focal plane of the condenser is imaged on to a quadrant photodiode. This method is well suited to experiments requiring high time resolution, as the high intensity of the focused laser reduces the problem of shot noise that arises with lower-intensity images.

Particles such as cells and latex spheres can be induced to rotate using electric fields, a technique termed electrorotation. This has been used as a non-invasive technique to characterize the dielectric properties of single micron-sized particles [8]. To induce electrorotation of a particle, a rotating electric field is required and this is usually generated using microelectrode structures. Characterization of the rate of rotation of a particle as a function of the applied frequency can be used to determine the dielectric properties of the particle. The technique has been used to provide information on the biophysical properties of cells $[9,10]$, and has also been used to characterize cell populations as a pre-requisite to separation using dielectrophoretic methods $[11,12]$.

Many species of bacteria propel themselves through aqueous media by means of helical flagellar filaments several microns long and $20-30 \mathrm{~nm}$ in cross-section $[13,14]$. These flagella are driven at their base by a tiny rotary motor embedded in the cell wall, which is driven by ions travelling down the electrochemical gradient that the cell generates across the cytoplasmic membrane. The precise mechanism of energy transduction in these motors is not known. In the case of Escherichia coli and many other species, the powering ions are $\mathrm{H}^{+}$(protons) whereas, in the case of alkalophilic and marine species such as Vibrio alginolyticus, rotation is driven by $\mathrm{Na}^{+}$ions. The flagella of $\mathrm{H}^{+}$-driven motors rotate at rates exceeding $100 \mathrm{~Hz}$, while the $\mathrm{Na}^{+}$-driven filaments of $V$. alginolyticus have been observed rotating at speeds up to $1700 \mathrm{~Hz}$.

In this paper we show that the back-focal-plane method can be used to measure electrorotation of polystyrene beads about $1 \mu \mathrm{m}$ in diameter, and also the rotation of $V$. alginolyticus flagellar motors. Single beads or bacterial cells were trapped and rotation speeds in excess of $1000 \mathrm{~Hz}$ measured, all within a few seconds. Thus the technique allows swift and simple measurement of rapid rotation rates of micronsized particles. Applications include characterization of the dielectric properties of microscopic particles and investigation of the torque-generating mechanism of the bacterial flagellar motor.

\section{Materials and methods}

\subsection{Optical trap}

Figure 1 shows a schematic diagram of the optical trapping microscope, which is custom built on an optical table. The beam from a diode-pumped solid-state laser of wavelength $1064 \mathrm{~nm}$ (Elforlight, Northamptonshire, UK) is attenuated by 


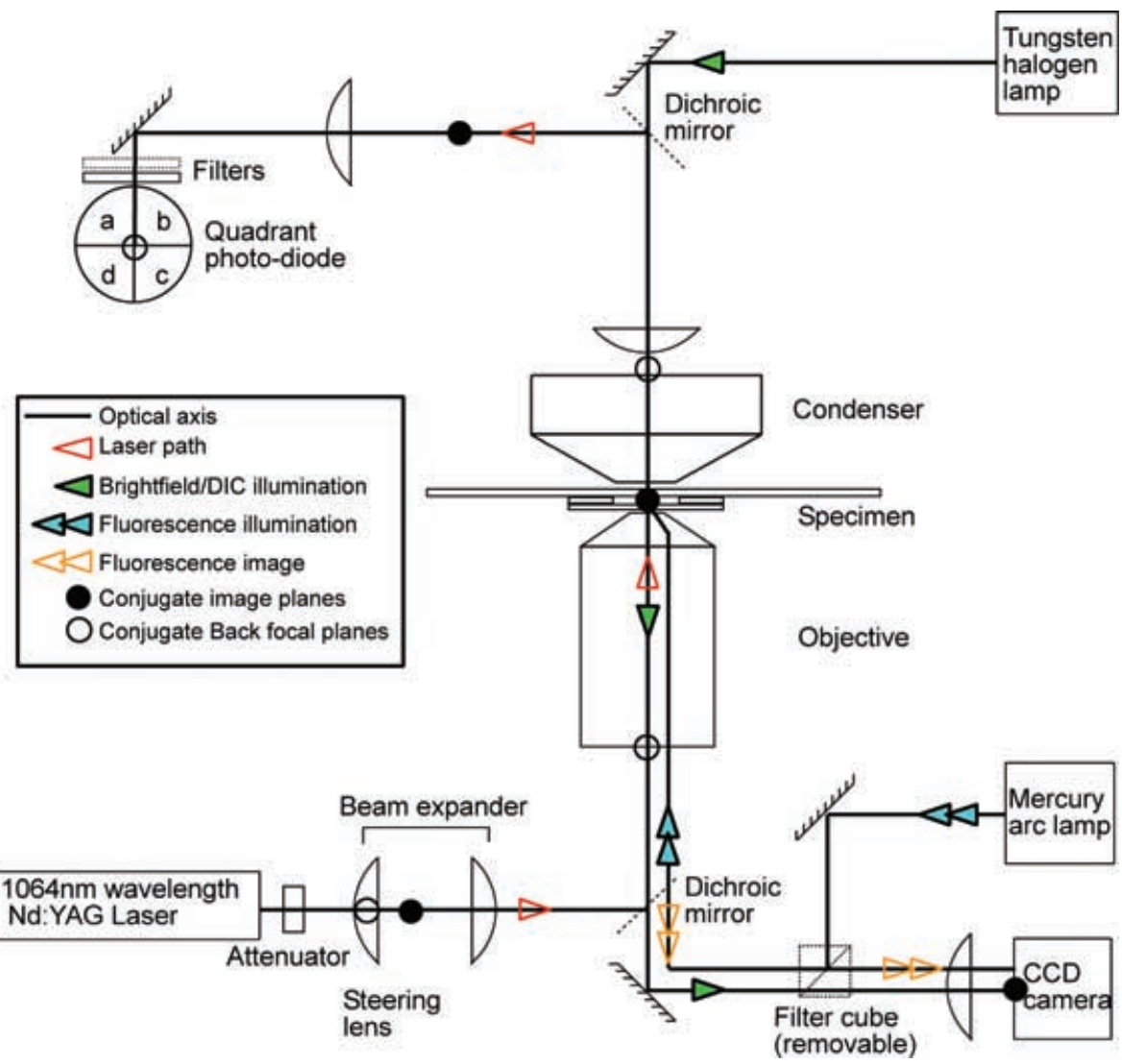

Figure 1. Schematic diagram of the optical trapping microscope: CCD, charge-coupled device. A near-infrared beam from a neodymium-doped yttrium aluminium garnet $(\mathrm{Nd}$ : YAG) laser is brought to a diffraction-limited focus by an oil immersion microscope objective of NA 1.4, forming the optical trap or 'tweezers'. The transmitted beam is recollimated by a condenser lens and projected on to a quadrant photodiode to form a position sensor. Illumination for bright-field or differential interference contrast (DIC) imaging is provided by a fibre-coupled tungsten-halogen bulb. Illumination for fluorescence is provided by a mercury arc lamp. Full circles indicate planes conjugate to the optical trap, and open circles indicate planes conjugate to the back focal plane of the objective.

a quarter-wave plate and polarizer and passed through a beam-expanding telescope to overfill slightly the back aperture of an oil immersion microscope objective of NA 1.4 (CFI Plan-fluor 100x, Nikon, Japan). The focused beam at the image plane forms the trap. The transmitted beam is recollimated by a condenser lens, and the condenser back focal plane is imaged to fill the surface $\left(78 \mathrm{~mm}^{2}\right)$ of a quadrant photo diode (Pin-SPOT 9DMI, UDT Sensors, California, USA). A long-pass filter (cut-off, $1000 \mathrm{~nm}$ ) is placed in front of the diode to block ambient light and a neutral density filter (ND 1) is added when the laser would otherwise saturate the diode. Tungsten-halogen illumination for bright-field or DIC imaging is filtered to remove short-wavelength light which damages biological specimens and is introduced via a multimode optical fibre. Epi-fluorescence illumination from a mercury arc lamp (Nikon, Japan) is introduced via a 
fluorescence filter cube (XF-108, Omega Optical, Vermont, USA). The image is projected on to a CCD video camera by a zoom lens.

\subsection{Data acquisition and analysis}

Photocurrents from each of the quadrants of the photodiode are amplified using a home-made current-to-voltage amplifier and sampled at up to $5 \mathrm{kHz}$ by a personal computer. The $x$ and $y$ coordinates of the deflection of a trapped particle in the focal plane of the microscope are calculated as

$$
\begin{aligned}
& X=\frac{(b+c)-(a+d)}{a+b+c+d}, \\
& Y=\frac{(a+b)-(c+d)}{a+b+c+d},
\end{aligned}
$$

where $a, b, c$ and $d$ are the sampled signals from the quadrant diode. The total magnitude of the signal $a+b+c+d$ may also be used as a measure of the displacement along the optical axis [15]. To obtain the rotation rate, $X$ and $Y$ are combined to form a complex signal $Z=X+\mathrm{i} Y$. For a particle rotating in a circle of radius $A$ at angular velocity $\omega_{0}, X=A \cos \left(\omega_{0} t\right), Y=A \sin \left(\omega_{0} t\right)$ and $Z=X+\mathrm{i} Y=$ $A \exp \left(\mathrm{i} \omega_{0} t\right)$ so that the Fourier power spectrum of $Z$, which indicates the intensity of each complex frequency $\omega$, will have a single peak at $+\omega_{0}$. Thus the power spectrum of $Z$ indicates both the speed and the direction of rotation [16].

\subsection{Polystyrene beads}

Polystyrene latex particles (beads) were obtained from Polysciences (Eppelheim, Germany), Interfacial Dynamics Corporation (Portland, Oregon, USA) and Molecular Probes (Eugene, Oregon, USA). Particles were plain polystyrene or modified with surface carboxyl or amino groups and ranged in size from 0.22 to $1.7 \mu \mathrm{m}$ in diameter. The rotation of perfectly spherical particles cannot be determined optically. To introduce asymmetry, larger particles were decorated with small numbers (e.g. three or four) of smaller particles. Covalent coupling of carboxy to amino beads was achieved by incubation with a 1-(3dimethylaminopropyl)-3-ethylcarbodiimide (EDC) cross-linker (Sigma, UK), while non-covalent hydrophobic coupling between plain polystyrene beads was accomplished by overnight incubation in $0.5-1.0 \mathrm{M} \mathrm{KCl}$, a medium of sufficient ionic strength to overcome the screening effects of electrical double layers at the beads' surfaces. For electrorotation experiments, particles were resuspended at a density of approximately $10^{5}-10^{6} \mathrm{ml}^{-1}$ in either $\mathrm{KCl}$ or in 4-(2-hydroxyethyl)-1pyre-azine ethanesulphonic acid (HEPES) buffer ( $\mathrm{pH} 7.0)$ of various conductivities.

\subsection{Erythrocytes}

Human blood was collected by venous puncture, erythrocytes harvested by centrifugation and washed three times in an iso-osmotic suspending medium $(9 \% \mathrm{w} / \mathrm{v}$ sucrose, $0.1 \% \mathrm{w} / \mathrm{v}$ glucose, $0.1 \mathrm{mM}$ ethylene diamine tetra acetic acid (EDTA), $0.8 \% \mathrm{w} / \mathrm{v}$ bovine serum albumin in diluted phosphate-buffered saline ( $\mathrm{pH} 7.4)$ ) with a conductivity of $36 \mathrm{mS} \mathrm{m}^{-1}$.

\subsection{Bacteria}

Frozen aliquots containing $100 \mu \mathrm{l}$ of the polar flagellated smooth-swimming $V$. alginolyticus strain NMB136 [17] were used to inoculate $3 \mathrm{ml}$ of growth medium 
(1\% polypeptone, $0.4 \% \mathrm{~K}_{2} \mathrm{HPO}_{4}, 3 \% \mathrm{NaCl}$ and $0.5 \%$ glycerol [18]). Cells were grown to late $\log$ phase (about $3 \mathrm{~h}$ ) at $30^{\circ} \mathrm{C}$ in a shaking incubator, harvested by centrifugation of $1 \mathrm{ml}$ samples at (2250-3500) $\times g$ for $2 \mathrm{~min}$, washed once and resuspended in motility medium containing $50 \mathrm{mM}$ Tris- $\mathrm{HCl}$, ( $\mathrm{pH}$ 7.5) (Sigma, UK), $5 \mathrm{mM}$ glucose and $5 \mathrm{mM} \mathrm{MgCl}_{2}$, and various concentrations of $\mathrm{NaCl} . \mathrm{KCl}$ was added such that the sum of $\mathrm{NaCl}$ and $\mathrm{KCl}$ concentrations was $300 \mathrm{mM}$. For observation and measurement of rotation, cells were diluted in motility medium to a density of approximately $10^{6} \mathrm{ml}^{-1}$ and added to a flow chamber constructed from a microscope slide, double-sided sticky tape and a coverslip. In media containing high concentrations of $\mathrm{Na}^{+}$, fast-swimming cells were allowed to swim into the trap. With low concentrations of $\mathrm{Na}^{+}$, slowswimming cells were caught in the trap by manually moving the microscope stage. The $X$ and $Y$ signals were fed into headphones worn by the experimenter, allowing immediate assessment of the signal from each cell that was trapped. Cells that were poorly motile or that were too symmetrical to give a strong signal were allowed to escape by shuttering the trap laser. Speeds were obtained by an algorithm that automatically detects peaks in spectra of $1 \mathrm{~s}$ blocks of data. Blocks in which the algorithm failed to detect a peak are not represented.

\subsection{Electrorotation}

Microelectrodes were fabricated on glass slides or coverslips by conventional photolithographic processes, and consisted of $100 \mathrm{~nm}$ of gold deposited over a $10 \mathrm{~nm}$ titanium adhesion layer. They had a gap of $25 \mu \mathrm{m}$ between opposing tips, as shown in figure $2(a)$. A four-phase direct digital synthesizer (DDS) was used to provide ac signals over a frequency range from $100 \mathrm{~Hz}$ to $20 \mathrm{MHz}$, at up to $12 \mathrm{~V}$ peak-to-peak $(\mathrm{p}-\mathrm{p})$. Custom-made electronic amplifiers were available to amplify this signal to a maximum of $60 \mathrm{~V} \mathrm{p}-\mathrm{p}$ in the frequency range from $10 \mathrm{kHz}$ to $5 \mathrm{MHz}$. The suspending medium conductivity was measured using a conductivity meter.

\section{Results}

Figure $2(b)$ shows a trapped bead held in the centre of the electrode array undergoing electrorotation. A schematic representation of the arrangement is shown in figure $2(c)$. The particle is a carboxy-modified polystyrene bead of diameter $0.83 \mu \mathrm{m}$, made optically anisotropic by labelling with fluorescent aminomodified polystyrene beads of $0.22 \mu \mathrm{m}$ diameter, and viewed with combined bright-field and fluorescence microscopy to show the large and smaller beads respectively. The four images are successive fields from a video sequence $(20 \mathrm{~ms}$ separation) and clearly show the bead rotating about the optical axis at approximately $10 \mathrm{~Hz}$. Figure $3(a)$ shows the $X$ and $Y$ signals from the quadrant detector sampled at $5 \mathrm{kHz}$ for a similar particle rotating at an angular velocity of $1062 \mathrm{~Hz}$, with an applied electrorotation voltage of $30 \mathrm{~V} \mathrm{p}-\mathrm{p}$ and a frequency of $2 \mathrm{MHz}$. The same data are plotted as $Y$ versus $X$ in figure $3(b)$. The power spectrum of $1 \mathrm{~s}$ of the combined signal $Z=X+\mathrm{i} Y$ is shown in figure $3(c)$, with the peak at $1062 \mathrm{~Hz}$ expanded in the inset. The figure shows that both the speed and the direction of rotation can be clearly determined from $1 \mathrm{~s}$ of data. This method, while particularly suitable for the measurement of rapid electrorotation of small particles, can also be applied to swift characterization of the electrorotation of larger particles such as mammalian cells. These particles are generally characterized at 
a)

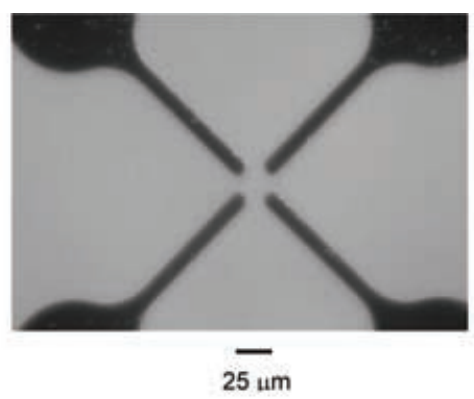

b)

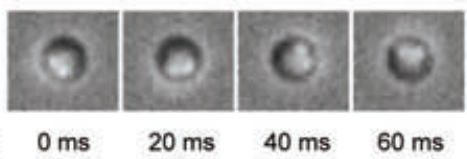

c)

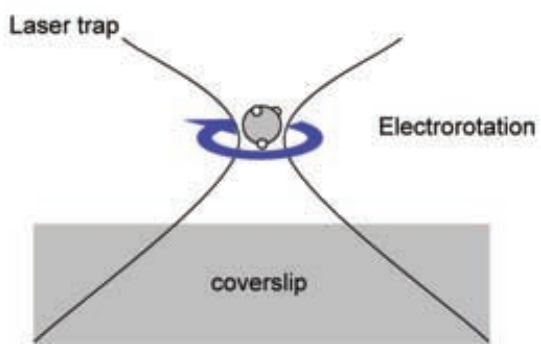

Figure 2. (a) Video image of a microelectrode array with a $25 \mu \mathrm{m}$ gap at the centre. (b) Successive images from a video sequence (captured at 50 images per second), showing electrorotation of a trapped bead. The large particle is carboxy-modified polystyrene latex, of diameter $0.83 \mu \mathrm{m}$, and is visible in bright-field illumination. Smaller particles (diameter, $0.22 \mu \mathrm{m}$ ) decorate the surface and are visible by virtue of being fluorescent. (c) In this figure the labelled particle rotates at about $10 \mathrm{~Hz}$ about the optical axis.

much lower rotation rates owing to the potentially adverse effect of high electric fields. As an example, figure $3(d)$ shows the $X$ signal (equivalent to figure $3(a)$ ) for a trapped human erythrocyte electrorotating at $3 \mathrm{~Hz}$, with an applied electric field frequency of $2 \mathrm{MHz}$ and $5 \mathrm{~V} \mathrm{p}-\mathrm{p}$ in a medium of conductivity $36 \mathrm{mS} \mathrm{m}^{-1}$.

A typical electrorotation spectrum for a carboxy-modified bead of $0.83 \mu \mathrm{m}$ diameter decorated with amino-modified beads of $0.22 \mu \mathrm{m}$ diameter is shown in figure 4. The figure shows two spectra for the same particle: one obtained with an applied voltage of $5 \mathrm{~V} \mathrm{p}-\mathrm{p}$ and the other obtained with $7 \mathrm{~V} \mathrm{p}-\mathrm{p}$. Electrorotation speed scales as the square of the voltages, as expected from theory, and the data clearly show a single peak characteristic of a solid dielectric particle such as a polystyrene particle [8]. The lines are fits to data using equation (3) (see discussion section). Fitting parameters are given in the figure caption. The high fitted value for the conductivity of the latex particle is due to mobile counter-charges in the double layer that screens surface charges and equates to a surface conductance of 
a)

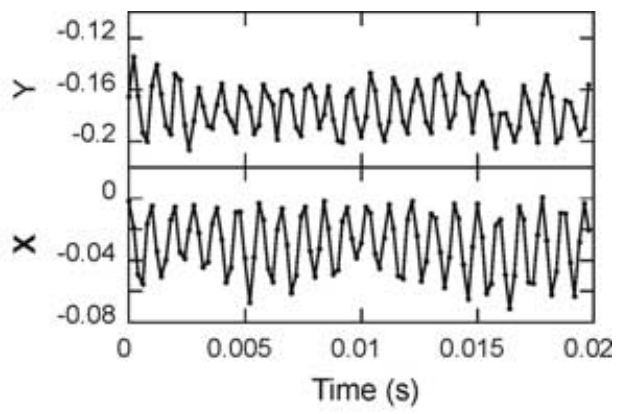

b)
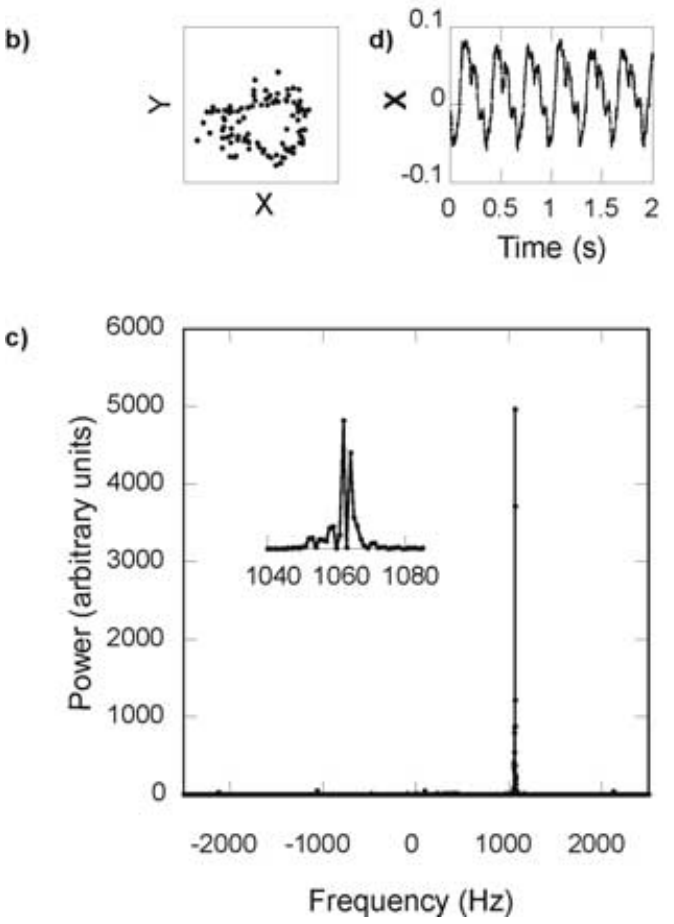

Figure 3. (a) $X$ and $Y$ signals sampled at $5 \mathrm{kHz}$ for a plain polystyrene bead of $1.0 \mu \mathrm{m}$ diameter decorated with plain polystyrene beads of $0.22 \mu \mathrm{m}$ diameter rotating at an angular velocity of $1062 \mathrm{~Hz}$. (b) The same data plotted as $Y$ versus $X$. (c) The power spectrum of $1 \mathrm{~s}$ of the combined signal $Z=X+\mathrm{i} Y$. The inset shows the peak at $1062 \mathrm{~Hz}$ with an expanded frequency scale. This spectrum was obtained for an applied voltage of $30 \mathrm{~V} \mathrm{p}-\mathrm{p}$ at a frequency of $2 \mathrm{MHz}$ with the particle held approximately $10 \mu \mathrm{m}$ above the centre of the electrode array on the coverslip. Particles were suspended in $1 \mathrm{mM}$ HEPES buffer ( $\mathrm{pH} 7.0$ ), with a conductivity of $1.5 \mathrm{mS} \mathrm{m}^{-1}$. (d) $X$ signal showing the electrorotation of an erythrocyte occurring at approximately $3 \mathrm{~Hz}$. The frequency was $2 \mathrm{MHz}$ and the applied voltage $5 \mathrm{~V} \mathrm{p}-\mathrm{p}$.

$1.5 \mathrm{nS}$. The spectrum was obtained by setting a suitable electric field frequency, recording the speed of rotation over a 1 or $2 \mathrm{~s}$ time interval and then moving on to the next field frequency. In this way a full spectrum could be obtained within about $1 \mathrm{~min}$. Clearly, this process could be made considerably faster by programming the DDS to sweep through a range of frequencies, recording $1 \mathrm{~s}$ or less of rotation data at each frequency. 
Figure 5(a) shows a schematic diagram of a $V$. alginolyticus cell held in the optical trap. Cells align with their long axis parallel to the optical axis, as the laser beam focus is also elongated along this axis. Examples of the $X$ and $Y$ signals are shown in figure $5(b)$, for a cell in a medium containing $50 \mathrm{mM} \mathrm{Na}^{+}$.

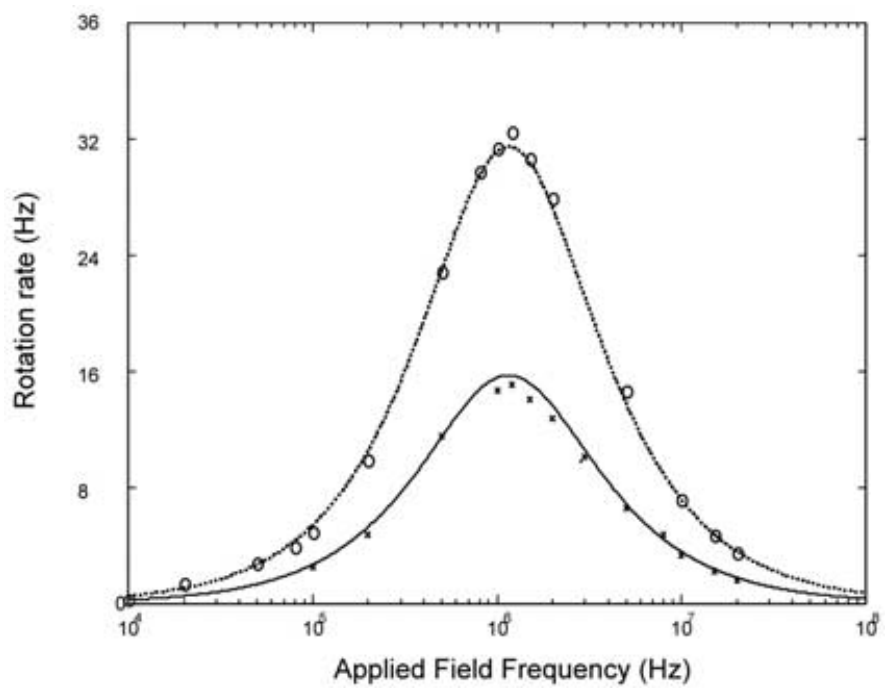

Figure 4. Electrorotation spectra for a carboxy-modified polystyrene particle of $0.83 \mu \mathrm{m}$ diameter labelled with $0.22 \mu \mathrm{m}$ amino-modified beads: $(\times)$, speeds obtained with an applied voltage of $5 \mathrm{~V} \mathrm{p}-\mathrm{p}$; (०), speeds obtained with $7 \mathrm{~V} \mathrm{p}-\mathrm{p}$; (一), (- - - -), best fits to the data using equation (3), with particle permittivity $\varepsilon_{\mathrm{p}}=2.5$ and particle surface conductance $K_{\mathrm{s}}=1.5 \mathrm{nS}$, equivalent to a particle conductivity $\sigma_{\mathrm{p}}=7.2 \mathrm{mS} \mathrm{m}^{-1}$.

a)

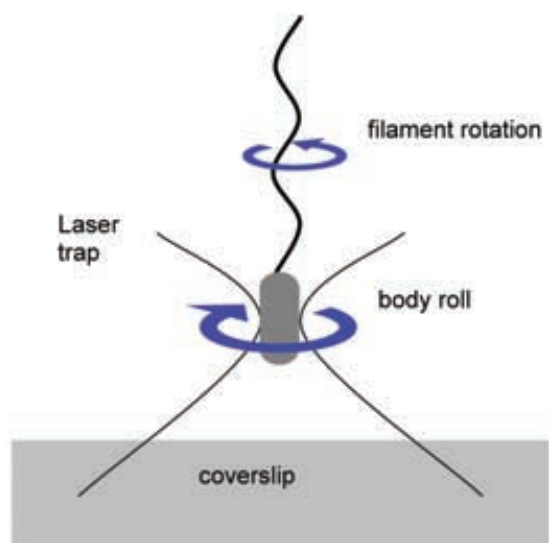

Figure 5. (a) Schematic diagram of a $V$. alginolyticus cell held in the optical trap. (b) $X$ and $Y$ signals from a trapped cell in a medium containing $50 \mathrm{mM} \mathrm{Na}^{+}$. The smallamplitude fast oscillation and large-amplitude slow oscillation are due to rotation of the flagellar filament and counter-rotation of the cell body respectively. $(c)$ The same data as $(b)$ plotted as $X$ versus $Y$. $(d)$ Power spectrum of $1 \mathrm{~s}$ of the combined signal $Z=X+\mathrm{i} Y$. The insets show filament and body-roll peaks on linear scales as in figure $3(c)$. The main figure uses a double logarithmic scale. Filament and body-roll rotations are in opposite directions, as indicated in $(a)$. The background noise in the spectrum is Lorentzian, characteristic of Brownian motion of a trapped particle. 
b)

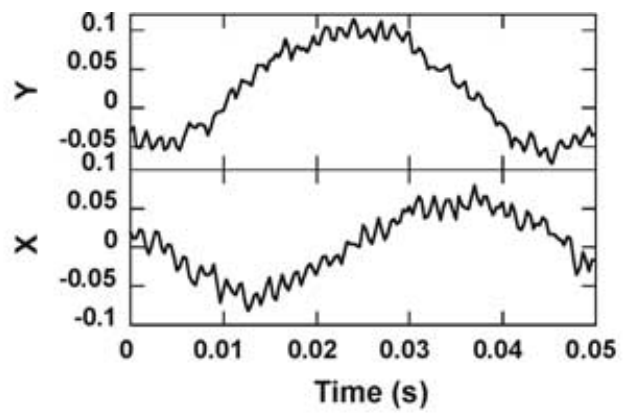

c)

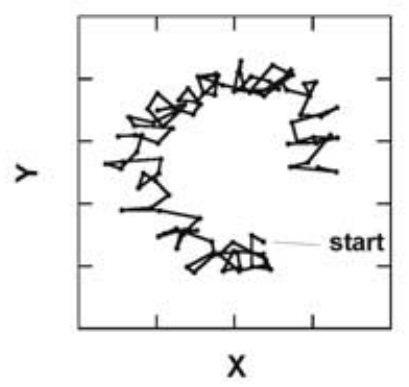

d)

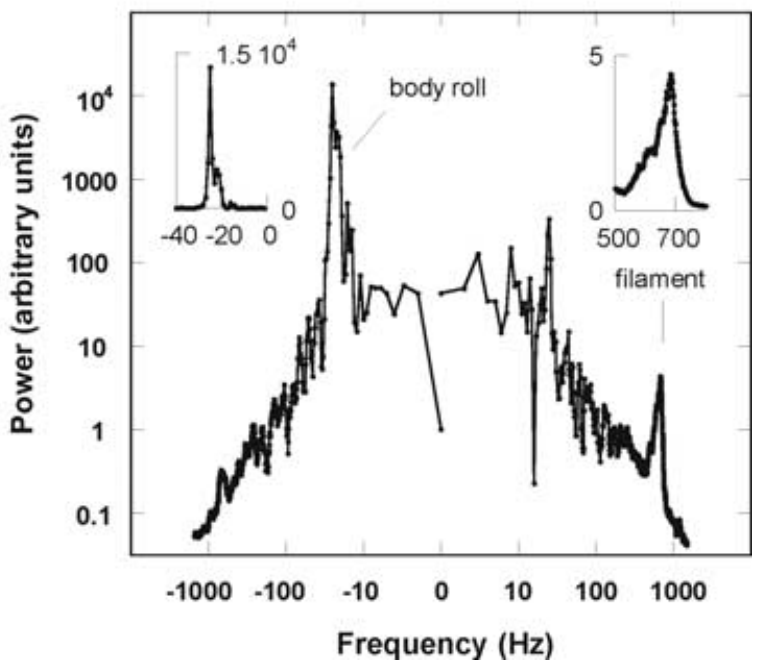

Figure 5. Continued.

The small-amplitude fast oscillation at about $700 \mathrm{~Hz}$ is due to rotation of the flagellar filament. It is unlikely that the filament itself, which is only $30 \mathrm{~nm}$ in diameter $[19,20]$, generates this signal. Most probably it is the wobble of the cell body caused by filament rotation that is detected. The slow oscillation at about $25 \mathrm{~Hz}$ is due to counter-rotation of the cell body. Counter-rotation occurs because there are no external torques acting on the cell. The torque generated by the motor is equal to the rotational speed divided by the drag coefficient for either the filament or the cell body, and the ratio of speeds is simply the inverse of the ratio of drag coefficients. Figure $5(c)$ shows the same data as figure $5(b)$ plotted as $Y$ versus $X$, and figure $5(d)$ shows the power spectrum of $1 \mathrm{~s}$ of the combined signal $Z=X+\mathrm{i} Y$. The insets in figure $5(d)$ show sections of the power spectrum in the 
a)

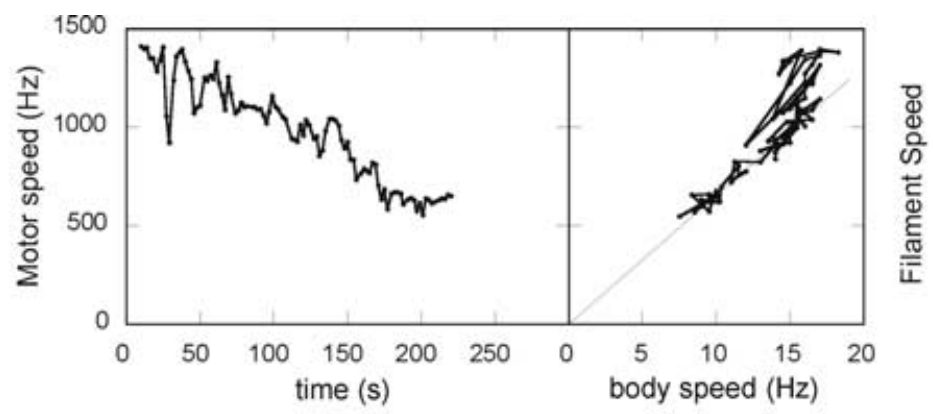

b)

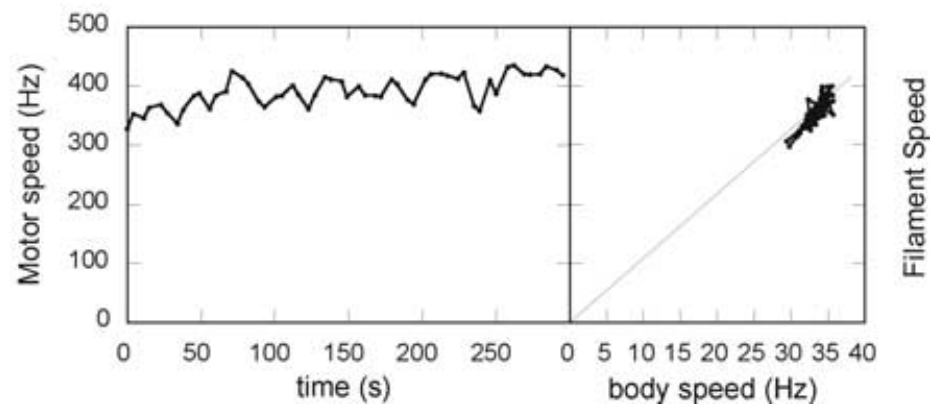

Figure 6. (a) On the left, motor speed versus time for a trapped $V$. alginolyticus cell in an aerobic medium containing $300 \mathrm{mM} \mathrm{Na}^{+}$. The motor speed is equal to the difference between body roll and filament speeds. The motor slows down owing to photodamage caused by the optical trap. On the right, filament speed versus body roll speed for the same cell. The line indicates a constant ratio of filament to cell body drag coefficients for the latter part of the data only. (b) As (a) but anaerobic and with $50 \mathrm{mM} \mathrm{Na}^{+}$. The initial speed is slower than in $(a)$ but does not decrease with time, indicating that oxygen is involved in the mechanism of photodamage.

vicinity of the body-roll (top left) and filament (top right) peaks, as in figure $3(c)$. Note the considerable difference in scales. The main plot of figure $5(d)$ shows the entire spectrum on a double logarithmic scale. It is clear from both figure $5(c)$ and figure $5(d)$ that the filament and body-roll rotations are in opposite directions, as expected.

The left-hand part of figure $6(a)$ shows the motor speed (equal to the sum of the absolute values of body roll and filament speeds) versus time for a trapped $V$. alginolyticus cell in an aerobic medium containing $300 \mathrm{mM} \mathrm{Na}^{+}$. The cell slows down owing to damage to the flagellar motor caused by the optical trap. The mechanism of photodamage is not fully understood but is thought to be mediated by excited-state (singlet) oxygen [21]. The right-hand part of figure $6(a)$ shows the filament speed versus body roll speed for the same cell. The line is drawn to guide the eye and demonstrates that filament and body roll speeds are proportional for data points after about $80 \mathrm{~s}$, indicating a constant ratio of drag coefficients. Before $80 \mathrm{~s}$, it appears that either the drag coefficient of the filament increases or the drag coefficient of the cell body decreases. Given the overall downward trend in speeds with time, the latter seems more likely. Possible reasons for changes in drag coefficients are discussed later. Figure $6(b)$ shows similar data to those of figure $6(a)$, but for a cell grown under anaerobic conditions and suspended in an anaerobic medium containing $50 \mathrm{mM} \mathrm{Na}^{+}$. The initial speed is considerably lower 
than in figure $6(a)$, partly because of the reduced sodium concentration [22]. Note also that this cell does not slow down with time, consistent with the hypothesis that oxygen is responsible for the photodamage seen in figure $6(a)$.

\section{Discussion}

\subsection{Electrorotation}

When a polarizable particle such as a latex bead or a biological cell is exposed to an externally applied electric field, a dipole moment is induced. If the electric field is non-uniform, this results in movement of the particle towards high- or lowstrength electric fields, a motion termed dielectrophoresis [23]. If the electric field is uniform, the dipole moment will align along the field direction. If the field changes direction, the induced dipole will attempt to realign itself with the field, that is, the particle experiences a torque. In a rotating electric field the particle is continuously attempting to align with the field, leading to rotation at a constant angular velocity. This is termed electrorotation.

The time-averaged torque on a particle is given by

$$
\Gamma_{\mathrm{ROT}}=-v \operatorname{Im}(\alpha)|\mathbf{E}|^{2},
$$

where $\mathbf{E}$ is the electric field, $v$ is the volume and $\alpha$ the effective polarizability of the particle [23]. In a viscous medium, a rotating particle experiences a drag torque, characterized by a friction coefficient, which depends on the viscosity $(\eta)$ of the medium and the geometry and surface properties of the particle. For laminar flow, the rotational frictional factor for a spherical stationary body, of radius $a$, rotating about its centre is

$$
f_{\mathrm{ROT}}=8 \pi \eta a^{3} .
$$

The angular velocity of the particle is

$$
R(\omega)=\frac{\Gamma_{\mathrm{ROT}}}{f_{\mathrm{ROT}}} .
$$

Substituting for the induced torque and the particle polarizability, the steady-state electrorotational angular velocity for a spherical particle is

$$
R(\omega)=-\frac{\varepsilon_{\mathrm{m}}|\mathbf{E}|^{2}}{2 \eta} \operatorname{Im}\left(\frac{\tilde{\varepsilon}_{\mathrm{p}}-\tilde{\varepsilon}_{\mathrm{m}}}{\tilde{\varepsilon}_{\mathrm{p}}+2 \tilde{\varepsilon}_{\mathrm{m}}}\right) .
$$

In this expression a complex dielectric constant is defined as $\tilde{\varepsilon}=\varepsilon-\mathrm{i} \sigma / \omega$, where $\sigma$ is the conductivity, $\varepsilon$ is the permittivity, $\omega$ is the angular frequency of the applied field and the subscripts $\mathrm{p}$ and $\mathrm{m}$ refer to the particle and medium respectively. For a latex particle the conductivity arises principally from charge movement in the double layer at the particle surface. This can be characterized by a surface conductance $K_{\mathrm{s}}$, which is related to particle conductivity $\sigma_{\mathrm{p}}$, and radius $a$ via $\sigma_{\mathrm{p}}=2 K_{\mathrm{s}} a$. Therefore, for a given value of surface conductance, the smaller the particle the larger is the effective conductivity. A solid homogeneous dielectric particle exhibits a single (Maxwell-Wagner) interfacial relaxation and consequently 
one rotation peak. Note that the rotation rate is independent of particle size but varies with the square of the electric field. However, the torque (as given by equation (1)) is determined by the particle volume. In both cases, maximum torque and maximum rotation rate occur at the characteristic interfacial relaxation frequency.

Combining electrorotation with laser tweezers as a particle manipulation and characterization tool provides several benefits [24-26]. Laser trapping can be used to select single cells of interest from the bulk population, bringing them into the centre of an electrode array for subsequent characterization. The laser trap also acts as a combined bearing-levitation system. Cells are prevented from adhering to the surfaces of the chamber and both cell-substrate interactions and near-wall hydrodynamic forces are eliminated. The action of the laser trap in holding the particle in a fixed position means that electrorotation spectra can be recorded over a wide range of frequencies and suspending medium conductivities, where other forces such as dielectrophoresis or electrohydrodynamic forces would otherwise cause the particle to move from the central field region of the electrode. This means that both higher voltages and much smaller electrode gaps can be used so that high speeds and torques can be generated if so desired.

In the early work of Fuhr et al. [27], dielectrophoresis was used to calibrate the force experienced by a particle in the optical trap. Schnelle et al. [25] combined laser tweezers with a dielectrophoretic field cage for single particle dielectric spectroscopy and showed how a cell could be moved inside the field cage to map the electric field distribution via the variation in rotation rate. The standard way of measuring the electrorotation of a particle has been by timing the rotation rate of an optically anisotropic particle with a stopwatch. Particles are rotated at very slow speeds (once or twice a second), often while resting on the glass bottom of a measuring chamber. Although reasonably accurate, this is a time-consuming method and does not lend itself either to measuring large numbers of cells or to determining temporal changes in cell behaviour. Cell-substrate interactions can also interfere with the rotation data, as can drift of the particle due to hydrodynamic or dielectrophoretic forces. In addition, the particle must be sufficiently optically anisotropic to be seen reliably by the naked eye.

In order to circumvent some of these problems, several automated approaches have been developed. Reichle et al. [28] developed a pinhole technique, introducing a small slot into the optical path and detecting temporal modulation in the light amplitude with a photodiode. One rotation spectrum covering 2.5 decades of frequency could be obtained in approximately $3 \mathrm{~min}$. This technique was elaborated upon by Mietchen et al. [26] who developed a virtual software masking method for analysing rotation data at speeds up to $7 \mathrm{~Hz}$. Rotation direction could be determined by ensuring that the software mask was asymmetric with respect to the direction of rotation. De Gasperis et al. [24] analysed digitized video recordings of a rotating cell using image-processing algorithms, while Zhou et al. [29] developed a computer-aided automatic imaging technique that could simultaneously measure the rotation of up to 256 particles. Both of these methods required intensive off-line image processing, so that Holzel [30] developed an online video processing system using a transputer-based moment generator enabling a complete spectrum (covering six decades of frequency) to be recorded every $3 \mathrm{~min}$. The back-focal-plane method described in this paper does not require sophisticated image processing techniques and can measure rotation rates from a 
few hertz to over $1 \mathrm{kHz}$, including direction of rotation. Through software control of the DDS frequency generator, it would be possible to obtain a complete electrorotation spectrum in a few tens of seconds.

\subsection{Bacterial flagellar motor}

Many different techniques have been used to study rotation of the bacterial flagellar motor [14]. For many years the dominant technique for observing single motors was the tethered cell assay, where a cell is attached to a microscope coverslip by a flagellar filament and the motor causes the whole cell to rotate at about $10 \mathrm{~Hz}$. Flagellar rotation is characterized by low Reynolds numbers, so that inertia can be ignored and the rotation rate is equal to the instantaneous torque divided by the rotational drag coefficient. The drag coefficient of the cell body is considerably greater than that of the filament, and thus the motor in a tethered cell operates in a low-speed high-load regime compared with the motor in a swimming or trapped cell. Various methods have been employed to observe faster flagellar rotation. The first was similar to the method that we report here but applied to a population of cells rather than a single cell [31]. The image of a chamber containing cells at a density of $4 \times 10^{8} \mathrm{ml}^{-1}$ was projected on to a position-sensitive photodiode, and the power spectrum of the diode signal showed body-roll and filament frequency peaks similar to those of figure $5(d)$, although considerably broader. Other techniques have used electrorotation to increase the speed of tethered cells [32-34], attached small polystyrene beads to truncated flagella [35] or observed rotating filaments directly using laser dark-field (LDF) microscopy [36]. These methods have shown that the torque generated by the flagellar motor is on the order of $3 \times 10^{-18} \mathrm{Nm}$ at stall, is nearly constant up to a speed of hundreds of hertz (dependent upon the temperature and the species of bacteria), falls through zero at its maximum natural rotation rate and resists further externally applied increases in rotation rate. If the motor is driven backwards, it resists with a torque similar to that which it generates at stall. The torque at stall is proportional to the electrochemical gradient that powers the motor. The speed of $\mathrm{Na}^{+}$-driven motors in swimming cells of $V$. alginolyticus increases with increasing $\mathrm{Na}^{+}$ concentration, although the extent to which this is due to any corresponding increase in the electrochemical gradient of $\mathrm{Na}^{+}$is not clear. These and other details of the mechanism of the flagellar motor have been reviewed by Berry and Armitage [14].

Assays in which cells are immobilized and rotation of a bead or of a flagellar filament is observed are slow and labour intensive. The time invested in finding a single cell to observe makes these methods suitable for extended observation of a single flagellar motor but less suitable for rapid measurements on many motors. As well as being fast and easy, the method reported here has the advantage that cells are essentially in their natural state, and the possibility of filament-surface interaction hindering rotation that is present in LDF experiments on immobilized cells is avoided. LDF microscopy has also been used to observe rotation of flagella in swimming cells [37], but in this technique each cell swims through the detector area in about $0.05 \mathrm{~s}$ or less, and thus yields very few data. Furthermore, in LDF microscopy, scattered light from the cell body causes a large flare in images which masks several microns of the filament, thus requiring the cell to have at least a $5 \mu \mathrm{m}$ flagellum before any useful signal can be obtained. 
The optical tweezers method requires the rotating particle to be anisotropic. With beads this was achieved by labelling with smaller beads, with cells this is unnecessary. In the case of the $V$. alginolyticus, the source of anisotropy is likely to be the cell itself. The cell body is not a perfect isotropic cylinder with hemispherical end caps but may bend slightly and may have asymmetric internal or external features. Also, the flagellar filament need not be placed exactly at the centre of the cell pole, in which case asymmetry is introduced if the rotation axis is different from the axis of the cell body. As with all biological specimens, there is considerable variation from cell to cell both in the magnitude of the signal and in the speed of rotation under a given set of conditions. Note also that for some cells the directions of filament and body-roll rotation were reversed. As strain NMB136 has a unidirectional polar flagellar motor, this indicates reversal of the orientation of the cell in the trap (see figure $5(a)$ ).

The origin of fluctuations in speed that lead to broadening of the spectral peaks in figures $3(c)$ and $5(d)$ is not certain. In the case of the flagellar motor, one possibility is speed fluctuations intrinsic to the motor itself. The possible origins and significance of these fluctuations have been discussed elsewhere [14]. However, Brownian motion will cause the orientation of any rotating object to fluctuate, and this will lead to speed fluctuations via changes in the rotational drag coefficient. In particular, deformation or deflection of the flagellar filament by Brownian motion is likely to contribute significantly to fluctuations in its drag coefficient.

The laser power used to trap specimens in this report varied up to as much as approximately $100 \mathrm{~mW}$. Thus the intensity of light at the specimen is extremely high, and the potential for photodamage or 'opticution' is also high. This damage may occur in several ways, through localized heating, two-photon absorption or the generation of free radicals. Photodamage by the trap laser limits observations on a single cell in aerobic conditions to a few minutes or less, depending on the extent of damage tolerated. The dependence of opticution upon laser wavelength, intensity and other factors has been measured using the flagellar motor of tethered E. coli cells as an indicator of damage [21]. That work indicates that photodamage is minimum at wavelengths of 820 and $980 \mathrm{~nm}$ and may be considerably reduced by removal of oxygen (as confirmed here). Scavengers of singlet oxygen may also be effective and have the advantage of being simpler to apply than an anaerobic technique.

Another limitation of the technique is that the density of cells must be high enough to allow a cell to be caught within a reasonable time, which increases the rate at which a second cell will swim into the trap. We plan to avoid this problem in future by conducting experiments in a flow cell with several adjacent streams of laminar flow. Cells will be collected from one stream and carried into other streams that contain no cells and different concentrations of $\mathrm{Na}^{+}$or other agents that affect the motor. This technique will allow rapid measurement of the effects of various media on flagellar rotation. By observing a single cell under a variety of conditions, variations due to individual differences between cells will be eliminated, allowing a detailed investigation of the effects of ion concentration and other agents upon flagellar rotation.

Experiments in which electrorotation is used to exert torque upon tethered cells are subject to problems arising from the large size and asymmetry of the tethered cell [34]. It should be possible in future to avoid these problems by 
applying a torque, using electrorotation, to spherical beads attached to the rotating flagellar stubs protruding from cell bodies which have been stuck to a surface. Their rotation can be measured with an optical trap [35], using the tweezers method described here to calibrate the electrorotation torque on the beads. From equation (2), a particle with a diameter of $1.0 \mu \mathrm{m}$ rotating at $1062 \mathrm{~Hz}$ indicates a torque of $2 \times 10^{-17} \mathrm{~N} \mathrm{~m}$. This is more than six times larger than the stall torque of the flagellar motor, and thus is adequate to explore the entire range of the torquespeed relationship. It should be noted that the torque or speed of a particle undergoing electrorotation is a function of both the applied field frequency (see figure 4) and the conductivity of the suspending medium. For a given particle size, maximum speed occurs when the imaginary part of the effective polarizability is maximum (cf. equation (3)). This can be achieved by using a particle with a high effective conductivity suspended in a medium of low conductivity. More torque is generated by large particles, but unfortunately the particle conductivity is inversely related to particle radius through the ratio $\sigma_{\mathrm{p}}=2 K_{\mathrm{s}} a$. In our experiments, high torque and speed were generated using beads of diameter about $1 \mu \mathrm{m}$ suspended in a low-conductivity buffer, such as $1 \mathrm{mM}$ HEPES. Whereas higher ionic strength is required for experiments on the flagellar motor of $V$. alginolyticus, $1 \mathrm{mM}$ HEPES is suitable for rotation experiments using bacterial species with $\mathrm{H}^{+}$-driven motors.

\section{Summary}

We have demonstrated a method that allows fast and easy measurement of rapid rotation of small particles, using optical tweezers and back-focal-plane position detection. We have demonstrated applications of the technique to obtaining electrorotation spectra for particle characterization, and to speed measurements of the bacterial flagellar motor.

\section{Acknowledgments}

Richard Berry would like to acknowledge the support of Wellcome Trust Research Career Development Fellowship (reference 053940). Hywel Morgan would like to acknowledge the award of a Royal Society-Leverhulme Senior Research Fellowship. We would also like to thank Bill Monaghan and Mary Robertson for fabricating the electrodes.

\section{References}

[1] Ashrin, A.Dziedzic, J. M.Bjorkholm, J. E.Chu, S., 1995, Opt. Lett., 11, 288.

[2] Svoboda, K.Block, S. M., 1986, A. Rev. Biophys. Biomol. Struct., 23, 247.

[3] Menta, A. D.Rief, M.Spudich, J. A.Smith, D. A.Simmons, R. M., 1994, Sci., 283, 1689.

[4] Simmons, R. M.Finer, J. T.Chu, S.Spudich, J. A., 1999, Biophys. J., 70, 1813.

[5] Berry, R. M.Berg, H. C., 1996, Proc. Natl. Acad. Sci. USA, 94, 14433.

[6] Allersma, M. W.Gittes, F.deCastro, M. J.Stewart, R. J.Schmidt, C. F., 1997, Biophys. J., 74, 1074.

[7] Lang, M. J.Asbury, C. L.Shaevitz, J. W.Block, S. M., 1998, Biophys. J., 83, 491.

[8] Arnold, W. M.Zimmermann, U., 2002, J. Electrostat., 21, 151.

[9] Hu, X.Arnold, W. M.Zimmermann, U., 1988, Biochim. Biophys. Acta, 1021, 191.

[10] Gimsa, J.Schnelle, T.Zechel, G.Glaser, R., 1990, Biophys. J., 66, 1244. 
[11] Becker, F. F., Wang, X. B., Huang, Y., Pethig, R., Vykoukal, J., and Gascoyne, P. R. C., 1994, Proc. Natl. Acad. Sci. USA, 92, 860.

[12] Chan, K. L.Morgan, H.Morgan, E.Cameron, I. T.Thomas, M. R., 1995, Biochim. Biophys. Acta, 1500, 313.

[13] Macnab, R. M., 1996, In Escherichia Coli and Salmonella: Cellular and Molecular Biology, edited by F. C. Neidhardt, R. Curtiss, I, J. L. Ingraham, E. C. C. Lin, G. Lowe, B. Magasanik, W.S. Reznikoff, M. Riley, M. Schaechter, and H. E. Umbarger (Washington, DC: American Society for Microbiology), pp. 123-145.

[14] Berry, R. M.Armitage, J. P., 2000, Adv. Microbial. Physiol., 41, 291.

[15] Pralle, A.Prummer, M.Florin, E.-L.Stelzaer, E. H. K.Horber, J. K. H., 1999, Microsc. Res. Technique, 44, 378.

[16] Berry, R. M.Turner, L.Berg, H. C., 1999, Biophys. J., 69, 280.

[17] Kojima, S.Atsumi, T.Muramoto, K.Kudo, S.Kawagishi, I.Homma, M., 1995, J. Molec. Biol., 265, 310.

[18] Asai, Y.Kojima, S.Kato, H.Nishioka, N.Kawagishi, I.Homma, M., 1997，J. Bacteriol., 179, 5104.

[19] Allen, R. D.Baumann, P., 1997, J. Bacteriol., 107, 295.

[20] Follett, E. A. C., 1971, J. Gen. Microbiol., 32, 235.

[21] Neuman, K. C.Chadd, E. H.Liou, G. F.Bergman, K.Block, S. M., 1963, Biophys. J., 77, 2856.

[22] Yoshida, S.Sugiyama, S.Hojo, Y.Tokuda, H.Imae, Y., 1999, J. biol. Chem., 265, 20346.

[23] Jones, T. B. 1995, Electromechanics of Particles (Cambridge University Press).

[24] De Gasperis, G.Wang, X. B.Yang, J.Becker, F. F.Gascoyne, P. R. C., 1990, Measmt. Sci. Technol., 9, 518.

[25] Schnelle, T.Muller, T.Reichle, C.Fuhr, G., 1998, Appl. Phys. B, 70, 267.

[26] Mietchen, D.Schnelle, T.Muller, T.Hagedorn, R.Fuhr, G., 2000, J. Phys. D, 35, 1258.

[27] Fuhr, G.Schnell, T. H.Müller, T.Hitzler, H.Monajembashi, S.Greulich, K.-O., 2002, Appl. Phys. A, 7, 385.

[28] Reichle, C.Muller, T.Schnelle, T.Fuhr, G., 1998, J. Phys. D, 32, 2128.

[29] Zhou, X.-F.Burt, J. P. H.Pethig, R., 1999, Phys. Med. Biol., 43, 1075.

[30] Holzel, R., 1998, Biochim. Biophys. Acta, 1425, 311.

[31] Lowe, G.Meister, M.Berg, H. C., 1998, Nature, 325, 637.

[32] Washizu, M.Kurahashi, Y.Iochi, H.Kurosawa, O.Aizawa, S.I., Kudo, S., Magariyama, Y., and Hotani, H., 1987, IEEE Trans. Ind. Applica., 29, 286.

[33] Berg, H. C.Turner, L., 1993, Biophys. J., 65, 2201.

[34] Berry, R. M.Berg, H. C., 1993, Biophys. J., 76, 580.

[35] Ryu, W. S.Berry, R. M.Berg, H. C., 1998, Nature, 403, 444.

[36] Kudo, S.Magariyama, Y.Aizawa, S.-I., 2000, Nature, 346, 677.

[37] Magariyama, Y.Sugiyama, S.Muramoto, K.Kawagishi, I.Imae, Y.Kudo, S., 1990, Biophys. J., 69, 2154. 